\title{
Non-Singular Thermodynamic Gravity
}

\author{
Pharis E. Williams \\ New Mexico Tech., Socorro, NM, USA
}

Received 2013-10-19; Revised 2013-12-13; Accepted 2013-12-23

\begin{abstract}
Newton formed his laws of motion, but still had to assume a law of gravity in order to determine the motions of bodies interacting through gravitational forces. Newton gave a prescription of how to find the dynamics produced by gravity. Einstein sought to determine the motions of gravity without using a force. Rather, he sought a system of equations by which the dynamics of bodies interacting through gravity were determined by the least distance between two points in a curved space. In both of these approaches gravity was basically independent of other forces of nature. Newton's approach was to provide a system of equations that determined the motion of bodies interacting through forces in any choice of geometry. Einstein's approach was to require that the force of gravity determine the geometry since he sought to find the motions without using a force. Newton gave three laws of motion in equations that could be used to find various types of energy such as potential energy or kinetic energy. This arrangement has the forces determining the energy of interactions. The approach presented here basically reverses these roles of energy and forces. It starts with a law stating the conservation of energy which states how any exchange of energy between a system and its surroundings affects the system's energy and the work it can perform. It is a law stating how these three energies, exchange energy, system energy and work, can interact. The expression for work, force acting through a distance, must be a path dependent term which also makes the exchange energy path dependent. However, the system's energy is not path dependent. The difference between the path dependence of the work and the path independence of the system's energy is shown to be crucial to the law's determination of dynamics.
\end{abstract}

Keywords: Gravity, Gauge Fields, Gauge Potentials, Fun Damental Particles, Non-Singular Potential

\section{INTRODUCTION}

The premise upon which this article is based is that the fundamental laws are sufficiently general to be used to determine both the forces that ultimately cause dynamic interaction and the dynamics that results from interactions.

Thus, the fundamental laws should be expected to provide the force laws, the equations of motion and whether the geometry in which to specify the dynamics may be freely chosen or if a particular geometry is required. Further, the fundamental laws will indicate whether the various forces such as gravitational and electromagnetic are independent or are inductively coupled. If the fundamental laws indicate that gravity is independent of other forces of nature then the other forces need not enter into an article on gravity alone. If the fundamental laws indicate an inductive coupling between the various forces of nature then any particular force may not be singled out for discussion by itself.

\section{THEREOTICAL FUNDATIONS}

\subsection{Fundamental Laws}

The first law, the conservation of energy, does not state how the dynamics must go. This means that the conservation of energy statement says nothing explicitly about time. Newton's laws of motion also did not explicitly involve time either. Time was, however, implicitly involved in Newton's laws as the acceleration, to which the force was related, was the time rate of change of velocity. The conservation of energy, 
however, does not even include time implicitly. This approach must then have a second law which tells how the dynamics must go. The adopted second law seems rather vague as its statement does not seem to depend upon time, but instead is a statement that in essence denies perpetual motion. The second law, as made by Caratheodory (1909), is a statement that surrounding any state point that a system may occupy there exists neighboring points to which the system may not go reversibly. That is if the system goes to these specific points and then returns to the original point it will be changed due to the round trip.

To summarize and simplify these adopted laws they are:

1st Law: Conservation of Energy Equation (1):

$$
\mathrm{dE}=\mathrm{dU}-\mathrm{F}_{\mathrm{i}} \mathrm{dx}^{\mathrm{i}}, \text { where } \mathrm{i}=1,2,3,4
$$

\section{2nd Law: Denial of Perpetual Motion}

Neighboring any state point there exist points that the system may not go to reversibly.

\subsection{Some Immediate Results}

Some immediate results of using these laws together become important later where gravity is concerned. Williams $(1997$; 2001; 2010a) derived one of the first immediate results to be that the 2 nd law guarantees the existence of an integrating factor for the 1st law that is a function of velocity only then Equation (2):

$$
\mathrm{dS}=\frac{\mathrm{dU}}{\phi}-\frac{\mathrm{Fdx}}{\phi}=\frac{\mathrm{dU}}{\phi}-\mathrm{fdx}
$$

This is important for two reasons. The first reason is that the existence of an integrating factor guarantees the existence of an absolute velocity, as was shown by Williams (1997). This is important since it provides a means of defining time. Plus, this method of defining time, since it relates time to space in an absolute fashion, provides a description of dynamics based upon absolute space and time. Secondly, the existence of the integrating factor guarantees the existence of another state function that can be obtained from the 1st law by multiplying the 1 st law by the integrating factor. This new state function, that may be called the entropy, as it is in thermodynamics, has several extremely important roles in the dynamics of the system. These roles include: (1) determining the flow of motion through the principle of maximum entropy for isolated systems which do not exchange energy with their surroundings, (2) determining the important distinction between the very stable constant entropy systems and those systems whose entropy is changing so as to seek a maximum and (3) determining the characteristic properties of bodies whose characteristics do not change as these bodies move around in space and time.

The third role of the entropy might well prove to be the most important role where trying to learn what gravity is as compared to how gravity causes dynamics, which is done by the first role. Bodies, or perhaps one might say fundamental particles, have various characteristics by which we describe them. For example, we talk of an electrically charged particle to mean that the particle possesses an electric charge. In the same manner we speak of a particle having a gravitational property through which the particle interacts with other particles. The standard model of physics assigns these characteristics to particles almost by assumption. The electrically charged particles are assumed to be those that have gauge potentials that obey the Maxwell equations of electromagnetism. Every particle is also assumed to have a gravitational potential through which it interacts with other gravitating particles. This third role of the entropy specifies the characteristics of particles that do not change as the particle moves through space and time. This means that it specifies what characteristics a particle must have if its entropy is to remain constant as the particle moves through space and time.

\section{RESULTS}

\subsection{Time and Physical Time}

In Newtonian dynamics time did not really have a definition and a common sense notion of time was used. There appeared two types of time in the relativistic theories of Einstein. The 'local' time played a role essentially like the role of time in Newtonian dynamics. A new type of time appeared when the relativistic theories introduced a differential element of space-time geometry. This differential element was called the proper time. Proper time was thus defined in terms of the local time and a geometric space.

The Dynamic Theory (DT), based upon the laws stated above, does not show a time in the statement of the laws. However, when the two laws are used together Williams $(2001 ; 2010 b)$ has shown that they require any dynamics to adhere to the stability conditions which are the second order derivatives of a set of energy functions with respect to a set of the independent variables. There is nothing specifying which variables are to be included in the set to be used. The 1st law consists of the differential of the system energy and differential path elements for each force applicable. This means that the number of 
variables required is the number of independent forces applicable plus one more. For three space forces as used in Newtonian dynamics there must be three plus one, or four independent variables. One is free to choose which set of four variables are to be used in the stability conditions. One convenient set of independent variables consists of the entropy and space variables. When this choice is made the stability conditions require that:

$$
\begin{aligned}
& \frac{\partial^{2} \mathrm{U}}{\partial \mathrm{S}^{2}}(\mathrm{dS})^{2}+2 \frac{\partial^{2} \mathrm{U}}{\partial \mathrm{S} \partial \mathrm{x}} \mathrm{dSdx}+\frac{\partial^{2} \mathrm{U}}{\partial \mathrm{x}^{2}}(\mathrm{dx})^{2}> \\
& 0-\frac{\left(\mathrm{m}_{\mathrm{o}} \mathrm{c}^{2}\right)^{2}}{\mathrm{U}^{3}}(\mathrm{dS})^{2}-2 \frac{\left(\mathrm{m}_{\mathrm{o}} \mathrm{c}^{2}\right)^{2} \mathrm{f}}{\mathrm{U}^{3}} \mathrm{dSdx} \\
& +\frac{\mathrm{m}_{\mathrm{o}} \mathrm{c}^{2}}{\mathrm{U}^{3}}\left[\mathrm{U}^{2} \mathrm{f}_{\mathrm{x}}-\mathrm{m}_{\mathrm{o}} \mathrm{c}^{2} \mathrm{f}^{2}\right](\mathrm{dx})^{2}>0
\end{aligned}
$$

This quadratic form must be positive definite and can be parameterized using the time and the absolute velocity, c, as:

$$
\begin{aligned}
& -\frac{\left(\frac{f_{o}}{m_{o} c^{2}}\right)^{2}}{U^{* 3}}(c d \tau)^{2}-2 \frac{\left(\frac{f_{o}}{m_{o} c^{2}}\right)}{U^{* 3}} f d x c d \tau- \\
& {\left[\frac{f^{2}}{U^{* 3}}-\frac{1}{U} * \frac{\partial f}{\partial x}\right](d x)^{2} \equiv(c d t)^{2}}
\end{aligned}
$$

Equation (4) uses and with Equation (5):

$$
\mathrm{U}^{*} \equiv \frac{\mathrm{U}}{\mathrm{m}_{0} \mathrm{c}^{2}}=\frac{1}{\phi} \quad \text { where } \phi \equiv \sqrt{1-\beta^{2}}
$$

Equation (3) uses the independent variables of space and entropy. Equation (4) defines time as a dependent variable. The units of the independent variable entropy has been converted to units of time in keeping with its role given by the 2 nd law as the measure of the flow of the dynamics created by the force. In this role it might be called the physical time, or the dynamic time, as its role is to establish how the dynamics must go as determined by the Entropy Principle.

One reason the set of independent variables of entropy and space becomes convenient is due to requirement that an isolated system which does not exchange energy with its surroundings must obey the Entropy Principle which requires that the differential of the entropy never be negative. This requires us to determine the expression for the differential of the entropy. For the simple case of a single force in the $\mathrm{x}$ - direction this expression for the differential of the entropy may be shown to be given by:

$$
\begin{aligned}
& \operatorname{cd} \tau=\left(\frac{\mathrm{m}_{\mathrm{o}} \mathrm{c}^{2}}{\mathrm{f}_{\mathrm{o}}}\right) \mathrm{U}^{* \frac{3}{2}} \\
& \left\{-\frac{\mathrm{f}}{\mathrm{U}^{* \frac{3}{2}}} \mathrm{dx}+\left[\left[\frac{2 \mathrm{f}^{2}}{\mathrm{U}^{* 3}}-\frac{1}{\mathrm{U}^{*}} \frac{\partial \mathrm{f}}{\partial \mathrm{x}}\right](\mathrm{dx})^{2}-(\mathrm{cdt})^{2}\right]^{\frac{1}{2}}\right\}
\end{aligned}
$$

Equation (6) shows several things that should be noted. The first is that the physical time, as denoted by $\tau$, does not differ from the time in the absence of a force. This means that a space-time manifold does not exist outside of the dynamics created by the force. This is also consistent with the notion that the physical time gives the flow of the motion. Another way of stating this is that within the DT a kinematic space-time, as developed in Einstein's relativistic theories, does not exist. One other thing should be noted here and that is the multiplicative factor that is common to all terms on the right hand side. Such a common factor on a manifold was named a gauge function by Weyl (1952) as he showed such a function acted to establish the distance measure, or gauge, of the manifold. Equation (6) shows it acts to set the gauge of the physical time for the isolated system.

\subsection{Stable, Isentropic States}

The common multiplicative term on the right hand side establishes a means of investigating the very stable states that occur when looking into constant entropy states. This also is the connection that allows the DT to apply the two fundamental laws into additional, interesting areas of physics. One area that it leads into is the area that should one be given an electrostatic gauge potential then an investigation of isentropic states leads to Schrodinger's quantum mechanics as was shown by London (1927). But what if one asks where the electrostatic potential is given? Does one need to assume Maxwell's electromagnetic field equations in addition to the 1 st and 2 nd laws? The same question might be asked concerning whether one also needs to assume a force law for gravity. Must a gravitational force law be assumed here just as Newton found a force law to be needed?

\subsubsection{Fundamental Particles}

In addition to asking what states might be possible given an electrostatic potential, there is another question 
that may be asked of the requirement imposed by isentropic states. This one turns the question around and asks, "What are the gauge potentials that may exist for isentropic states where these gauge potentials do not change as they are moving around in space and time? This basically is asking if the isentropic requirement establishes the characteristics of fundamental particles. If it does than the gauge function is the starting point for this investigation. However, we are more concerned with the first and second order derivatives of the gauge function.

Before going further with this line of discussion we must return to the 1st law and ask how many independent work terms must be considered in a study of natural phenomena. Of course we know of the possibility of three independent forces in each of the three space directions. We also know of a fourth force when considering thermodynamic systems. If this force were independent of the three space forces we would have four independent forces and our expression for the physical time would depend upon four force variables and one time variable. This would also mean that our gauge function is based upon five independent variables and there would be five gauge potentials since the gauge potentials are the first order derivatives of the gauge function.

Any integrable geometric manifold of any number of independent variables must satisfy the Bianchi conditions and these conditions establish interrelationships between the second order derivatives of the gauge function. When there are four independent variables, three of space and one of time, these relationships among the second order derivatives are the Maxwell equations of electromagnetism. Williams (2010c) has shown that for five independent variables additional equations are specified by the Bianchi conditions for a total of eight different equations relating the second order derivatives of the gauge function. There are five gauge potentials. When the time derivative of the gauge function is taken the result is the electrostatic potential. The three space derivatives of the gauge function produce the three vector potentials. However, it is the fifth gauge potential that is the subject of this investigation. This gauge potential turns out to be the gravitational potential and if the isentropic condition specifies the characteristics of the gauge potentials that a particle may have that does not change as it moves around in space and time it is this fifth gauge potential that will specify the gravitational character of the particle.

It may be instructive to present the five variable field equations derived by Williams $(2001 ; 2010 d)$. First, the five variable fields may be presented in matrix form as Equation (7):

$$
\mathrm{F}_{\mathrm{ij}}=\left[\begin{array}{ccccc}
0 & \mathrm{E}_{1} & \mathrm{E}_{2} & \mathrm{E}_{3} & \mathrm{~V}_{4} \\
-\mathrm{E}_{1} & 0 & \mathrm{~B}_{3} & -\mathrm{B}_{2} & \mathrm{~V}_{1} \\
-\mathrm{E}_{2} & -\mathrm{B}_{3} & 0 & \mathrm{~B}_{1} & \mathrm{~V}_{2} \\
-\mathrm{E}_{3} & \mathrm{~B}_{2} & -\mathrm{B}_{1} & 0 & \mathrm{~V}_{3} \\
-\mathrm{V}_{4} & -\mathrm{V}_{1} & -\mathrm{V}_{2} & -\mathrm{V}_{3} & 0
\end{array}\right]
$$

The field equations are Equation (8):

$$
\begin{array}{cc}
\bar{\nabla} \overline{\mathrm{B}}=0 & \frac{1}{\mathrm{c}} \frac{\partial \overline{\mathrm{B}}}{\partial \mathrm{t}}+\bar{\nabla} \times \overline{\mathrm{E}}=\overline{0} \\
\bar{\nabla} \times \overline{\mathrm{B}}-\frac{1}{\mathrm{c}} \frac{\partial \overline{\mathrm{E}}}{\partial \mathrm{t}}+\mathrm{a}_{0} \frac{\partial \overline{\mathrm{V}}}{\partial \gamma}=\frac{4 \pi \overline{\mathrm{J}}}{\mathrm{c}} & \bar{\nabla} \times \overline{\mathrm{E}}+\mathrm{a}_{0} \frac{\partial \mathrm{V}_{4}}{\partial \gamma}=4 \pi \rho \\
\frac{\partial \rho}{\partial \mathrm{t}}+\bar{\nabla} \overline{\mathrm{J}}+\mathrm{a}_{0} \frac{\partial \mathrm{J}_{4}}{\partial \gamma}=0 & \bar{\nabla} \times \overline{\mathrm{V}}+\mathrm{a}_{0} \frac{\partial \mathrm{B}}{\partial \gamma}=0 \\
\bar{\nabla} \mathrm{V}_{4}+\frac{1}{\mathrm{c}} \frac{\partial \overline{\mathrm{V}}}{\partial \mathrm{t}}=\mathrm{a}_{0} \frac{\partial \overline{\mathrm{E}}}{\partial \gamma} & \bar{\nabla} \overline{\mathrm{V}}+\frac{1}{\mathrm{c}} \frac{\partial \mathrm{V}_{4}}{\partial \mathrm{t}}=-\frac{4 \pi}{\mathrm{c}} \mathrm{J}_{4}
\end{array}
$$

\subsubsection{Quantization}

Isentropic states may exist in two ways. The physical time may be identically zero or it may swing positive and then negative but must always return to zero periodically like a sine wave. The isentropic condition, in this fashion, quantizes the gauge potentials with a quantum number. This is important as it establishes the quantized electric charge on fundamental particles. This is also important as it establishes a distinction between different fundamental particles.

\subsubsection{Gravity}

The steps in the process of determining the gauge requirements of the isentropic condition are many and tedious. However, the process starts with the quantization of the gauge potentials and then forming the fields from these quantized potentials and ensuring that the gauge function and its derivatives satisfy all of the field equations. After this is done the logarithm of the square root of the gauge function is given by:

$\ln \mathrm{f}^{\frac{1}{2}}=\left(\frac{\mathrm{r}_{\mathrm{o}}}{\mathrm{r}}\right) \mathrm{e}^{-\left(\frac{\lambda_{\mathrm{N}}}{\mathrm{r}}\right)} \mathrm{e}^{-\mathrm{H}_{\mathrm{o}} \mathrm{t}} \mathrm{e}^{-\mathrm{K}_{\gamma} \gamma}$, with $\mathrm{K}_{\gamma}=\frac{\mathrm{H}_{\mathrm{o}}}{\mathrm{a}_{\mathrm{o}} \mathrm{c}}$

Equation (9) uses $H_{o}$ as Hubble's constant and $a_{0} c$ as a constant pertaining to the limiting rate of mass conversion.

From this gauge function the fields become: 


$$
\begin{aligned}
& \frac{\mathrm{F}_{\mathrm{ij}}}{\left(\frac{\mathrm{e}}{4 \pi \varepsilon_{0}}\right)} \approx \\
& \begin{array}{ccccc}
\mathrm{Z}\left(\frac{\mathrm{r}_{\mathrm{o}} \mathrm{H}_{\mathrm{o}}}{\mathrm{cr}^{2}}\right)\left(1-\frac{\lambda_{\mathrm{N}}}{\mathrm{r}}\right) \mathrm{e}^{-\left(\frac{\lambda_{\mathrm{N}}}{\mathrm{r}}\right)} & 0 & 0 & 0 \\
-\mathrm{Z}\left(\frac{\mathrm{r}_{\mathrm{o}} \mathrm{H}_{\mathrm{o}}}{\mathrm{cr}^{2}}\right)\left(1-\frac{\lambda_{\mathrm{N}}}{\mathrm{r}}\right) \mathrm{e}^{-\left(\frac{\lambda_{\mathrm{N}}}{\mathrm{r}}\right)} & 0 & 0 & -\mathrm{Ze} \frac{\mathrm{a}_{\mathrm{o}} \mathrm{c}}{\mathrm{H}_{\mathrm{o}}} \mathrm{r}_{\mathrm{o}} \mathrm{K}_{\gamma}\left(\frac{1}{\mathrm{r}^{2}}\right)\left(1-\frac{\lambda_{\mathrm{N}}}{\mathrm{r}}\right) \mathrm{e}^{-\frac{\lambda_{\mathrm{N}}}{\mathrm{r}}} \\
0 & 0 & 0 & 0 \\
0 & \mathrm{Ze} \frac{\mathrm{a}_{\mathrm{o}} \mathrm{c}}{\mathrm{H}_{\mathrm{o}}} \mathrm{r}_{\mathrm{o}} \mathrm{K}_{\gamma}\left(\frac{1}{\mathrm{r}^{2}}\right)\left(1-\frac{\lambda_{\mathrm{N}}}{\mathrm{r}}\right) \mathrm{e}^{-\frac{\lambda_{N}}{\mathrm{r}}} & 0 & 0 & 0 \\
0 & 0 & 0
\end{array}
\end{aligned}
$$

Equation (9) and (10) show that the potentials and the fields are non-singular in that they return to zero as $r$ tends to zero. This feature of the electric and the gravitational fields is very different from the classical $1 / r^{2}$ character from Maxwell's electromagnetism and Newton's gravity.

An interesting feature of this process for finding the characteristics of fundamental particles includes the ability to theoretically form the ratio of the electromagnetic and the gravitational forces to get Equation (11):

$$
\mathrm{F}_{\text {ratio }} \approx \frac{\mathrm{H}_{\mathrm{o}}^{2}}{\mathrm{a}_{\mathrm{o}}^{2} \mathrm{c}^{2} \mathrm{~K}_{\gamma}^{2}}=\frac{\frac{\mathrm{e}^{2}}{4 \pi \varepsilon_{\mathrm{o}}}}{\mathrm{Gm}^{2}}
$$

Which is, of course, the experimentally measured ratio of the electromagnetic and the gravitational forces.

\subsubsection{Theoretical Summary}

In the preceding two fundamental laws were adopted and used to show that they require a certain form of a gauge function for all particles that do not change their gauge characteristics as they move around in space and time. The gauge potentials of these unchanging fundamental particles show gauge properties known to exist for most particles. For example, they possess long range electrostatic and gravitational potentials that diminishes with distance as $1 / \mathrm{r}$. They also possess long range forces that vary as $1 / \mathrm{r}^{2}$. However, these gauge potentials also possess some properties not previously considered for fundamental particles. Two of these new properties are the time dependence of the gravitational potential and the non-singularity of both the electrostatic and gravitational potentials which requires that these potentials vanish as $\mathrm{r}$ tends to zero.
With respect to gravity these two new properties of the gravitational potential produce many new gravitational predictions that agree with previously obtained data such as planetary orbital perihelion advancement, cosmological red shifts, dark matter and dark energy. In addition to the new properties of the gauge potentials of fundamental particles, the inductive coupling between the electromagnetic and the gravitational fields provide new predictions of physical phenomena that have either already been measured or may be measured.

\subsubsection{Comparison between Theory and Experiment}

The following is a partial list of phenomena where predictions from the new theoretical properties of gravity have been compared to existing experimental data. While there also exists many ways of comparing the new theoretical properties of the electrostatic potential to existing and not-yet-obtained data this article will only address those involving the gravitational properties.

\subsection{Perihelion Advance}

The gravitational force differs from the Newtonian gravitational force through the influence of the nonsingular character of the potential and the time. This nonsingular deviation depends, therefore, upon the $\lambda$ in the multiplicative exponential function. Williams (2010e) has shown that this deviation predicts an advancement of the perihelion of planetary orbits. This deviation is found by comparing the frequency of small radial oscillations about steady circular motion for the non-singular potential with the frequency of revolution. This produces a perihelion advance of:

$\delta \theta \cong 2 \pi\left(\frac{3 \lambda \mathrm{GMm}^{2}}{\mathrm{~L}^{2}}\right)$ 
Equation (12) predicts an advance that compares to the Einstein prediction when $\lambda=\mathrm{GM} / \mathrm{c}^{2}$.

\subsection{Red Shifts}

Williams (2010f) showed that the time dependence of the gravitational potential predicts a red shift in light received from distant stars. This calculation is done assuming isentropic propagation of light and yields a wave length shift of:

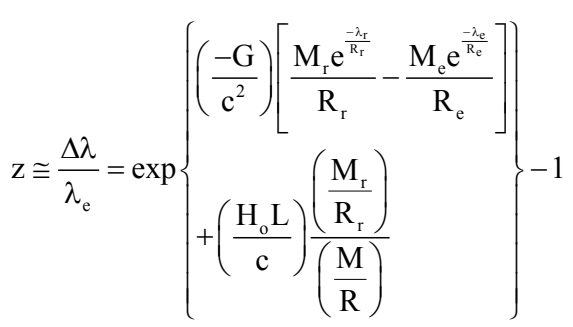

Equation (13) uses $\mathrm{M}$ and $\mathrm{R}$ without subscripts to refer to the mass and radius of the Earth. If the reception is done at the Earth's surface the ratio of the gravitational potential at reception and the Earth's surface cancels each other. This factor occurs in the expression for the red shift because our standard for comparison is the frequencies measured at the Earth's surface.

\subsection{Einstein's Field Equations}

Einstein sought to describe dynamics in terms of motion along the geodesic of a curved space rather than motion due to the action of a force. Though he determined a set of field equations that, in the limit, tend toward Newtonian gravitational dynamics, these field equations lend themselves to multiple solutions that depend upon various restrictive assumptions. The two adopted in the DT are such that one may choose the geometry in which to write the 1st Law and then apply the 2 nd Law to determine the dynamics. These laws were extended into the five variables of space, time and mass density before using them to determine the either the path required given some gauge potential or the particle characteristic that would be independent of the path.

Einstein only considered systems in which the mass was conserved. When mass is conserved in the five variable system Williams $(2010 \mathrm{~g})$ showed the effect is that of embedding a four variable hyper surface into the five variable chosen geometry of the five variable manifold. The procedure is to write the five variable manifold's energy-momentum tensor in terms of the hyper surface metric and then impose the restrictive assumption that mass be conserved. The surface energy- momentum tensor may now be found within the space tensor and written Equation (14):

$$
\mathrm{T}^{\alpha \beta}=\mathrm{T}_{\mathrm{sp}}^{\alpha \beta}-\frac{1}{\mathrm{c}^{2}}\left[\mathrm{~F}_{4}^{\alpha} \mathrm{F}^{4 \beta}-\frac{1}{2} \mathrm{~h}^{\alpha \chi} \mathrm{F}^{4 v} \mathrm{~F}_{4 v}\right]
$$

The form of this expression for the hyper surface energy-momentum tensor suggests writing:

$$
\mathrm{CT}^{\alpha \beta} \equiv \mathrm{G}^{\alpha \beta} \equiv \mathrm{R}^{\alpha \beta}-\frac{1}{2} \mathrm{~g}^{\alpha \beta} \mathrm{R}
$$

which, of course, look like the field equations of Einstein's general theory of relativity. Equation (15) are taken to be Einstein's field Equation (16):

$$
\mathrm{G}^{\mu \nu}=-\frac{8 \pi \kappa}{\mathrm{c}^{2}} \mathrm{~T}^{\mu v}
$$

where, is the gravitational constant.

This shows that there two ways to determine the dynamics within the DT. One method is to use the gravitational gauge potential in the five variable manifold of the 1st Law and seek to maximise the entropy for an isolated system when mass is conserved. The second method is to the equations of geodesics on the four variable hyper surface. However, in this case the coefficients in the energy-momentum tensor of the hyper surface can be determined from the given gauge potentials and, therefore, the solutions to the field equations are limited by the character of the gauge potentials. The nature of the field equations are such that for simple situations, such as when all potentials vanish except the gravitational potential, it easier to determine the dynamics using the non-zero potential in the five variable manifold while conserving the mass.

\subsection{No Big Bang}

The non-singular character of the gravitational potential predicts that there exists a point in time in the history of the universe where the expansion velocity of the universe was zero. Williams (2010h) showed at that point in time the universe was not at a point of singularity but was still of finite size and possessed nonzero expansion acceleration. Therefore, the non-singular gravitational potential denies the existence of a big bang beginning of the universe. To see the difference between the cosmologies of the classical, singular gravitational force and the time dependent, non-singular force the cosmological equation may be developed using the nonsingular potential to arrive at: 


$$
\frac{\mathrm{d}^{2} \mathrm{R}}{\mathrm{dt}^{2}}=\frac{4 \pi \mathrm{G} \rho_{\mathrm{o}}}{3}\left(1-\frac{\lambda}{\mathrm{Rr}}\right) \frac{\mathrm{e}^{-\frac{\lambda}{\mathrm{Rr}}} \mathrm{e}^{-\mathrm{H}_{\mathrm{o}} \tau}}{\mathrm{R}^{2}}
$$

Equation (17) shows that the scale factor of the universe, $\mathrm{R}$, experiences zero acceleration when $\mathrm{Rr}=\lambda$. Then when $\operatorname{Rr}>\lambda$ by only a slight amount the scale factor experiences an exponentially large acceleration until it becomes large enough to pull down the acceleration by the cubic factor in the denominator. Thus, the scale factor of the universe shows a decreasing acceleration as $\mathrm{R}$ gets larger, but never reverses into a deceleration.

Equation (17) also shows that the non-singular potential does not support a cosmological singularity when $r=0$ as would be required for a big bang model of the universe.

\subsection{Dark Matter}

Williams (2010i) solved the system of equations showing the time dependence of the gravitational field gives rise to the prediction that stars in the outer arms of spiral galaxies possess tangential velocities that correspond to the greater gravitational field strength of the center of the galaxy at a previous point in time corresponding to the distance the star is from the center of the galaxy divided by the speed of light. Thus, the tangential velocities do not fall off as predicted by Newtonian gravity.

When one uses the time dependent, non-singular gravitational field in the equations of motion for uniform circular motion one must account for the time delay for the gravitational time dependent change of the galactic central gravitational mass to travel to the radius at which one desires to calculate. This calculation results in the acceleration compared to the Newtonian acceleration to be given by a multiplicative factor reflecting the time dependence and the initial rate of change of the physical time when the gravitational effect leaves the galactic center $\dot{\tau}_{\mathrm{o}}$. The relation is:

$$
\begin{aligned}
\mathrm{a} & =\mathrm{a}_{\mathrm{N}}\left\{1-\frac{\mathrm{H}_{\mathrm{o}}^{2} \lambda}{2 \mathrm{r}} \mathrm{t}^{2}-\mathrm{H}_{\mathrm{o}} \dot{\tau}_{\mathrm{o}} \mathrm{t}\right\} \\
& \cong \mathrm{a}_{\mathrm{N}}\left\{1+\mathrm{H}_{\mathrm{o}} \dot{\tau}_{\mathrm{o}} \frac{\mathrm{r}}{\mathrm{c}}\right\}
\end{aligned}
$$

In the absence of a means of theoretically evaluating the initial conditions we may turn to experimental results. We now use the data that shows the acceleration begins to deviate from Newtonian when the acceleration drops to a value of $1.2 \times 10^{-10} \mathrm{~m} / \mathrm{sec}^{2}$ so that Equation (19):
$\mathrm{a}_{\mathrm{N}}=\frac{\mathrm{GM}}{\mathrm{r}_{\mathrm{c}}^{2}} \cong 1.2 \times 10^{-10} \Rightarrow \mathrm{r}_{\mathrm{c}} \cong \sqrt{\frac{\mathrm{GM}}{1.2 \times 10^{-10}}}$

Then requiring Equation (20):

$$
\dot{\tau}_{\mathrm{o}}=\frac{\mathrm{c}}{\mathrm{r}_{\mathrm{c}} \mathrm{H}_{\mathrm{o}}}
$$

Sets a value of $\dot{\tau}_{\mathrm{o}}$ in keeping with the data. Equation (18) becomes the approximation Equation (21):

$$
\mathrm{a} \cong \mathrm{a}_{\mathrm{N}}\left\{1+\frac{\mathrm{r}}{\mathrm{r}_{\mathrm{c}}}\right\}
$$

where, we see the short range Newtonian acceleration and the long range acceleration predicted by MOND.

It should be noted that the approximate linearity of the tangential velocity with respect to time displays an independence of the time it takes for light to travel from the galaxy to Earth. This apparent independence of time masks the fact that the gravitational strength of the galaxy, relative to the current epoch, depends upon the time of light travel to Earth.

\subsection{Dark Energy}

The time dependence of the gravitational field was shown by Williams (2010j) to change the prediction of the Chandrasekhar limiting mass. This time variation of the Chandrasekhar limiting mass prevents the use of the Type Ia supernovas as a standard candle with a constant luminosity. The change in the limiting mass with respect to universe time was shown to be given as Equation (22):

$$
\frac{\mathrm{dM}_{\mathrm{L}}}{\mathrm{d} \tau}=\left(\frac{9 \mathrm{H}_{\mathrm{o}}}{4}\right) \mathrm{M}_{\mathrm{Ch}}\left(1-\mathrm{H}_{\mathrm{o}} \tau\right)^{-\frac{13}{4}}
$$

The universe expansion factor may be taken from general relativity whose first integration is the Friedman equation. Equation (17) may again be used. When the initial conditions are evaluated to account for all mass and radiation energy the square of the universe expansion rate is given in terms of the red shift as Equation (23):

$$
\left(\frac{\dot{a}}{\mathrm{a}}\right)^{2}=\mathrm{H}_{\mathrm{o}}^{2}\left\{\left[\begin{array}{l}
\Omega_{\mathrm{M}} \mathrm{z}+\Omega_{\mathrm{M}} \frac{12-2 \mathrm{z}}{(3+2 \mathrm{z})} \\
+\Omega_{\mathrm{M}} \frac{\mathrm{z}(7-2 \mathrm{z})}{(3+2 \mathrm{z})}
\end{array}\right]+\Omega_{\mathrm{RO}}\right\}
$$

where, the $\Omega_{\mathrm{M}}$ varies as $(1+\mathrm{z})^{3}$. 


\subsubsection{Experiments with Inductive Coupling}

The gauge fields of the fundamental laws are inductively coupled. This means that the gravitational and the electromagnetic fields are not totally independent fields. Each is tied to the other. There are several experiments that may display this inductive coupling. This list is only representative of the possible experiments that use the inductive coupling of these fields.

\subsection{Radiation Energy Density and Pressure}

Williams (2010k) has shown that the inductively coupled gravitational and electromagnetic fields predict a radiation energy density given by the sum of the squares of the electromagnetic and gravitational field components as in Equation (24):

$$
\xi \equiv \frac{1}{8 \pi}\left[\overline{\mathrm{E}} \bullet \overline{\mathrm{E}}+\overline{\mathrm{B}} \bullet \overline{\mathrm{B}}+\overline{\mathrm{V}} \bullet \overline{\mathrm{V}}+\mathrm{V}_{4}^{2}\right]
$$

where, the $\mathrm{V}$ represents the gravitational vector field and the $\mathrm{V}_{4}$ is the gravitational potential. However, the radiation pressure is given by the sum of the squares of the electromagnetic components minus the square of the gravitational component or Equation (25):

$$
p=-\left(\frac{1}{24 \pi}\right)\left[E^{2}+B^{2}+3 V_{4}^{2}-V^{2}\right]
$$

Therefore, the radiation pressure will always be a little less than the radiation energy density. This is just what the Nichols and Hull experiment showed. It is true that the difference was less that the expected experimental error, but new experiments using interferometer techniques may be used to reduce the experimental error below that of the Nichols and Hull experiment.

The difference in the expression for the radiation energy density and the radiation pressure gives rise to the case where the pressure must be zero. This would be the case in space where no pressure can be supported due to the absence of inertial material. Since radiation pressure is the difference between the sum of the squares of the electromagnetic field components and the square of the gravitational field then the point of zero radiation pressure still requires that the radiation energy density be non-zero. If the Nichols and Hull data is used to estimate a value for the constant $a_{o}$ then the temperature of the zero pressure radiation may be determined. Initial estimates place the temperature in the low single digit temperatures.

\subsubsection{Cosmic Background Radiation}

As mentioned in the section on radiation energy density of the inductively coupled gauge fields consists of the addition of the squares of the components of the electromagnetic and gravitational fields. These results come from solutions to the wave equations that were developed from the field equations by Williams (20101). The totality of these waves include three electric field components, three magnetic field components, three gravitational field components and a gravitational potential for a total of ten wave components. The solution of the wave equations includes two types of waves. The first wave type is the transverse wave where the vector field components are perpendicular to the direction of wave propagation while the second wave type may be called nontransverse as none of the field components are perpendicular to the direction of wave travel.

The transverse wave solution consists of three components rather than the two components of standard electromagnetic theory. The third component is a gravitational field component which is directed opposite to the electric field component and contains only a small part of the wave energy when compared to the energy contained in the electric and magnetic components. This is the reason for the small difference between the radiation energy density and pressure. The non-transverse wave also consists of three components which are an electric field component, a magnetic field component and a gravitational potential component. This wave is rather peculiar in that it consists of two vector components and one scalar component. If all three components were vector components directed parallel, or anti-parallel, with the direction of wave travel, the wave might best be referred to as a longitudinal wave. However, since one wave component is a scalar component it would be misleading to call the wave a longitudinal wave.

Several predictions have been made using the Electromagnetogravitic (EMG) wave solutions, but these may be best addressed in other formats. What may be most useful herein is to present the influence of gravity upon EMG waves. The first influence has already been discussed in the immediately preceding sections on radiation energy density and pressure and the cosmic background radiation. Two other influences may be useful to mention here.

\subsection{Alternate Communications}

All of our communications systems that depend upon wave propagation utilize the transverse wave type. However, one may also use the non-transverse waves to communicate between two distant locations. The major problem in using the non-transverse waves to communicate is that a means of creating and detecting these waves must be found. The transverse and the nontransverse wave solutions appear as independent waves in the wave equations. However, when a transverse wave 
strikes a material interface, such as between air and glass, at an oblique angle some of the transverse wave energy may be converted into non-transverse wave energy depending upon the orientation of the electric field component with respect to the normal to the interface. Similarly, a non-transverse wave may have some of its energy converted into transverse wave energy when it strikes a material interface a certain way.

The design of a means of converting transverse wave energy into non-transverse wave energy constitutes a non-transverse wave sending antenna. Alternatively, a means of converting non-transverse wave energy into transverse wave energy constitutes a non-transverse wave receiving antenna. Designs of both the sending and receiving non-transverse wave antennas were completed by Williams $(2010 \mathrm{~m})$, but have not been tested.

\subsection{Neutrinos}

Neutrinos have the interesting property that they can pass through any material almost at will almost without being detected. There is an interesting similar property that involves the non-transverse wave. In transverse waves the electric field may accelerate electrons in a dipole antenna because the electric field is directed perpendicular to the direction of wave propagation. However, the electric field in a non-transverse wave is in the direction of propagation and this prevents the electric field from accelerating a free electron. This allows the non-transverse wave to propagate virtually without attenuation through materials that would stop a transverse wave. The skin depth for a non-transverse wave in copper has been calculated to be some $1010 \mathrm{~m}$. This sort of skin depth makes stopping a non-transverse wave as difficult as a neutrino.

Another feature of this theory upon gauge potentials lies in the fact that isentropic states may be found just as the section above showed that a fundamental particle may have certain quantized electrostatic potential properties. There are, however, five gauge potentials within this theory. In addition to the electrostatic potential there exist three vector potential components just as are seen in standard electromagnetism. Should one investigate an isentropic propagation of these vector potential one finds their energy to be quantized as $\varepsilon=$ $\mathrm{N}^{2} h v$ of course this quantized energy is similar to Einstein's quantized photon energy except that this energy displays the quantum number. But there is still another gauge potential in the theory and this is the gravitational potential. When Williams (2010n) investigated isentropic propagation of this potential he found that the energy is also quantized as $\varepsilon_{n}=N^{2} h v$.
The subscript has been used to indicate that this energy is the energy of a non-transverse particle which, because of the large skin depth of the nontransverse wave, behaves similar to the neutrino.

\subsection{Earth's Magnetic Moment}

Williams (2010o) has shown that the inductive coupling between the electromagnetic and gravitational fields leads to the prediction that an electrically neutral mass will have a magnetic moment. A theoretical determination of the inductive coupling constant gives a charge to mass ratio of $\beta=\sqrt{4 \pi \varepsilon_{0} G}$ where the dielectric and gravitational constants combine to give the inductive coupling constant. If this constant is used the magnetic moment of the Earth is predicted to be $8.6 \times 10^{22}$ amp- $\mathrm{m}^{2}$ instead of the experimentally determined $8.1 \times 10^{22}$ amp- $\mathrm{m}^{2}$. This is a rather amazingly accurate prediction given that it was made using the assumption of a uniform mass density for the Earth.

\subsection{Direct Measurement of the Coupling Constant}

Northrop Grumman hired a researcher at Carnegie Melon University to measure the coupling constant. This researcher, Dr. Dennis Shure, devised an experiment that dropped an electrically neutral mass, such as pure water among other types of neutral mass, through a sensitive toroid coil and measured the electrical signal as the mass went through the coil. The data showed the inductive coupling constant to be the $\beta$ above as predicted. This result has not yet been accepted for publication.

\subsection{Earth Flyby Anomalies}

Flyby anomalies have been recognized for decades and many good references may be found. What is to be considered here is the phenomenological formula:

$$
\begin{aligned}
\frac{\Delta \mathrm{v}_{\infty}}{\mathrm{v}} & =\mathrm{K}\left(\cos \delta_{\mathrm{i}}-\cos \delta_{\mathrm{f}}\right) \\
\mathrm{K} & =\frac{2 \omega_{\mathrm{e}} \mathrm{R}_{\mathrm{e}}}{\mathrm{c}}=3.099 \times 10^{-6}
\end{aligned}
$$

Equation (26) displays the initial and final declination angles. These angles appearing in the formula prompted the authors to ask about the potential of declination producing a physical effect.

The Dynamic Theory, due to its five variable basis, predicts an inductive coupling between the gravitational and the electromagnetic fields. This inductive coupling was shown to be Equation (27): 


$$
\beta=\sqrt{4 \pi \varepsilon_{0} G}
$$

where, $\varepsilon_{0}$ is the dielectric constant and $G$ is the gravitational constant. Williams $(2010 \mathrm{o})$ shown that this coupling predicts the magnetic moment of the Earth as Equation (28):

$$
\mu=\left(\frac{\mathrm{q}_{\text {eff }}}{2 \mathrm{M}}\right) I \omega_{\mathrm{e}}
$$$$
q_{\text {eff }}=\beta \mathrm{M}
$$

Where:

$\mathrm{M}=$ The mass of the Earth

$\mathrm{I}=$ The Earth's moment of inertia

$\mathrm{q}_{\text {eff }}=$ The effective electric charge due to the mass of the Earth

The magnetic force on a satellite with velocity, $\overline{\mathrm{v}}$ is given by Equation (29):

$$
\overline{\mathrm{f}}=\mathrm{q}(\overline{\mathrm{v}} \times \overline{\mathrm{B}})
$$

The magnetic field associated with a magnetic moment of a sphere is given by:

$$
\overline{\mathrm{B}}=\frac{\mu_{\mathrm{o}}}{4 \pi} \frac{\left[3 \overline{\mathrm{r}}(\bar{\mu} \bullet \overline{\mathrm{r}})-\bar{\mu} \mathrm{r}^{2}\right]}{\mathrm{r}^{5}}
$$

Equation (28) including the magnetic moment becomes:

$$
\bar{\mu}=\left(\frac{\mathrm{q}_{\text {eff }}}{2 \mathrm{M}}\right) \overline{\mathrm{I}} \omega_{\mathrm{e}}=\frac{\beta}{2} \frac{2 \mathrm{MR}^{2}}{5} \hat{\mu} \omega_{\mathrm{e}}=\frac{\beta \mathrm{MR}^{2} \omega_{\mathrm{e}}}{5} \hat{\mu}
$$

To find a change in velocity one may look at the change of momentum due to an impulse, or:

$\Delta \overline{\mathrm{v}}=\frac{\Delta \overline{\mathrm{p}}}{\mathrm{m}}=\int \frac{\mathrm{q}}{\mathrm{m}}(\overline{\mathrm{v}} \times \overline{\mathrm{B}}) \mathrm{dt}$

The effective charge for the satellite is:

$\mathrm{q}_{\text {eff }}=\beta \mathrm{m}$

Using Equation (30), (31) and (33) in Equation (32) obtains Equation (34):

$$
\begin{aligned}
\Delta \overline{\mathrm{v}} & =\int \beta \frac{\mu_{\mathrm{o}}}{4 \pi \mathrm{r}^{5}} \frac{\beta M R^{2} \omega_{\mathrm{e}}}{5}\left(\overline{\mathrm{v}} \times\left[3 \overline{\mathrm{r}}(\hat{\mu} \bullet \overline{\mathrm{r}})-\hat{\mu} \mathrm{r}^{2}\right]\right) \mathrm{dt} \\
& =\frac{\beta^{2} \mathrm{M} \mu_{\mathrm{o}} \mathrm{R}^{2} \omega_{\mathrm{e}}}{20 \pi} \int \frac{1}{\mathrm{r}^{3}}(\overline{\mathrm{v}} \times[3 \hat{\mathrm{r}}(\hat{\mu} \bullet \hat{\mathrm{r}})-\hat{\mu}]) \mathrm{dt} \\
& =\frac{2 \pi \varepsilon_{\mathrm{o}} \mu_{\mathrm{o}} \mathrm{GMR}^{2} \omega_{\mathrm{e}}}{20 \pi} \int \frac{1}{\mathrm{r}^{3}}(\overline{\mathrm{v}} \times[3 \hat{\mathrm{r}}(\hat{\mu} \bullet \hat{\mathrm{r}})-\hat{\mu}]) \mathrm{dt} \\
& =\frac{2 \mathrm{GMR}^{2} \omega_{\mathrm{e}}}{20 \mathrm{c}} \int \frac{1}{\mathrm{r}^{3}}(\overline{\mathrm{v}} \times[3 \hat{\mathrm{r}}(\hat{\mu} \bullet \hat{\mathrm{r}})-\hat{\mu}]) \mathrm{dt}
\end{aligned}
$$

It should be noted that the speed of light appears in the denominator because the dielectric constant of the charge to mass ratio, $\beta$, combines with the magnetic permeability.

Satellites in hyperbolic orbits have the radial position in the orbital plane given by Equation (35):

$r=\frac{a\left(\varepsilon^{2}-1\right)}{(1+\varepsilon \cos \phi)}$

Where Equation (36):

$\mathrm{a}=\left|\frac{\mathrm{K}}{2 \mathrm{E}}\right|$ where $\mathrm{K}=-\mathrm{GMm}$

And Equation (37):

$\varepsilon=\frac{1}{\cos \alpha}$ where $\alpha \equiv \frac{\pi-\Theta}{2}$

With $\Theta$ being the deflection angle. The angular momentum is found to be given by Equation (38):

$\mathrm{L}=\mathrm{mr}^{2} \dot{\phi}$

Equation (33) may be differentiated with respect to time to get the radial component of the velocity as Equation (39):

$\dot{\mathrm{r}}=\frac{\mathrm{dr}}{\mathrm{dt}}=\frac{\varepsilon \mathrm{r}^{2} \sin \phi}{\mathrm{a}\left(\varepsilon^{2}-1\right)} \dot{\phi}=$

$\frac{\varepsilon r^{2} \sin \phi}{\mathrm{a}\left(\varepsilon^{2}-1\right)} \frac{\mathrm{L}}{\mathrm{mr}^{2}}=\frac{\varepsilon \mathrm{L} \sin \phi}{\mathrm{ma}\left(\varepsilon^{2}-1\right)}$

While the azimuthal component of the velocity is Equation (40):

$$
\mathrm{v}_{\phi}=\mathrm{r} \dot{\phi}=\frac{\mathrm{L}}{\mathrm{mr}}
$$

Therefore, the total velocity in the orbital plane is:

$$
\begin{aligned}
\bar{v} & =\frac{\varepsilon L \sin \phi}{m a\left(\varepsilon^{2}-1\right)} \hat{r}+\frac{L}{m r} \hat{\phi}+0 \hat{\theta} \\
& =\frac{\varepsilon L \sin \phi}{m a\left(\varepsilon^{2}-1\right)} \hat{r}+\frac{L[1+\varepsilon \cos \phi]}{m a\left(\varepsilon^{2}-1\right)} \hat{\phi}+0 \hat{\theta} \\
& =\frac{L}{m a\left(\varepsilon^{2}-1\right)}\{\varepsilon \sin \phi \hat{r}+[1+\varepsilon \cos \phi] \hat{\phi}+0 \hat{\theta}\} \\
& =\frac{L \sqrt{\varepsilon^{2}+1+2 \varepsilon \cos \phi}}{m a\left(\varepsilon^{2}-1\right)}\left\{\begin{array}{l}
\frac{\varepsilon \sin \phi}{\sqrt{\varepsilon^{2}+1+2 \varepsilon \cos \phi}} \hat{r} \\
+\frac{[1+\varepsilon \cos \phi]}{\sqrt{\varepsilon^{2}+1+2 \varepsilon \cos \phi}} \hat{\phi}+0 \hat{\theta}
\end{array}\right\} \\
& =\frac{L \sqrt{\varepsilon^{2}+1+2 \varepsilon \cos \phi}}{m a\left(\varepsilon^{2}-1\right)} \hat{v}
\end{aligned}
$$


Now we may substitute Equation (41) into Equation (34) to get Equation (42):

$$
\begin{aligned}
& \Delta \overline{\mathrm{v}}=\frac{2 \mathrm{GMR}^{2} \omega_{\mathrm{e}}}{20 \mathrm{c}} \int \frac{1}{\mathrm{r}^{3}}\left(\begin{array}{c}
\frac{\mathrm{L} \sqrt{\varepsilon^{2}+1+2 \varepsilon \cos \phi}}{\mathrm{ma}\left(\varepsilon^{2}-1\right)} \\
\hat{\mathrm{v}} \times[3 \hat{\mathrm{r}}(\hat{\mu} \bullet \hat{\mathrm{r}})-\hat{\mu}]
\end{array}\right) \mathrm{dt} \\
& \left.=\frac{2 \mathrm{GMR}^{2} \omega_{\mathrm{e}}}{20 \mathrm{c}} \frac{\mathrm{L}}{\mathrm{ma}\left(\varepsilon^{2}-1\right)} \frac{\mathrm{mr}^{2}}{\mathrm{~L}} \int^{\frac{\sqrt{\varepsilon^{2}+1+2 \varepsilon \cos \phi}}{\mathrm{r}^{3}}} \mathrm{~d} \times[3 \hat{\mathrm{r}}(\hat{\mu} \bullet \hat{\mathrm{r}})-\hat{\mu}]\right) \\
& =\frac{\mathrm{GMR}^{2} \omega_{\mathrm{e}}}{10 \mathrm{ca}^{2}\left(\varepsilon^{2}-1\right)^{2}} \int_{[1+\varepsilon \cos \phi](\hat{\mathrm{v}} \times[3 \hat{\mathrm{r}}(\hat{\mu} \bullet \hat{\mathrm{r}})-\hat{\mu}])}^{\sqrt{\varepsilon^{2}+1+2 \varepsilon \cos \phi}} \mathrm{d} \phi
\end{aligned}
$$

Equation (42) has the qualitative features of the phenome-nological formula of Equation (26) in that it has the same coefficient that depends upon the Earth's rotation rate and the speed of light. The speed of light enters into the equation because the effective charge of both the masses is used. The vector products left in the brackets show that the final value of the change in velocity depends upon the angle of the orbit with respect to the Earth's magnetic moment. Since the Earth's magnetic North pole is nearly the normal to the equatorial plane, the dot product and the cross product may be seen to vanish for a satellite whose orbit is strictly in the equatorial plane. This argues that the satellite must have an orbit with some inclination in order to show a change in velocity. Equation (42) shows that for various orbits the change in velocity might be positive or negative as has been measured. Also, since R is a major fraction of $r$ the overall magnitude of the bracketed portion will lie within the zero to 2 value of the difference of declinations.

Only an integration of an actual satellite path will show final validity of the ability of Dynamic Theory to predict the anomalous velocities measured, however, the correct qualitative features are there and the quantitative value appears to be within the ballpark.

\section{CONCLUSION}

The fundamental laws adopted in the Dynamic Theory were those of classical thermodynamics and, therefore, have not yet been shown to invalidated by experiment. This article has presented the logical route that may be used to derive many experimental predictions in the realms of the atomic, nuclear and cosmological physics. However, concentration has been on gravitational aspects that best support the intended subject of this issue regarding Space Science.

There is one subject developed by Williams (2010p) that perhaps should also have been included and that is a solid state fusion reactor that could provide power and perhaps propulsion for space craft. This reactor concept has a patent pending and is a very compact form of a long term energy source.

\subsection{Gravity}

The theoretical foundation in thermodynamics lends itself to seeking answers to physical phenomenon not addressable by other current theories. For example, within this article the presentation has been limited to isolated systems that do not exchange energy with their surroundings. Then when one investigates the extremely stable isentropic states within these isolated systems one finds that this stipulation determines the characteristics that a fundamental particle may have if that particle's characteristics are to remain fixed as the particle moves around in space and time. These characteristics were found to be given by a gauge function which depended upon space, time and mass. The derivatives of this gauge function gave the gauge potentials and from these potentials one obtained the forces. The derivative of the gauge function with respect to time gave the electrostatic potential. The derivatives of the gauge function with respect to space variables gave the vector potentials. Finally, the derivative of the gauge function with respect to mass produced the gravitational potential.

The negative gradient of this gravitational potential produces the gravitational force. However, the gauge potentials are not the singular potentials of classical electromagnetism and Newtonian gravitation. Rather, these potentials are dependent upon space, time and mass in such a way that they are all non-singular. Also, while the electrostatic potential is time independent, the gravitational potential is time dependent. It is the inductive coupling of these potentials and the time dependent, non-singular character of the gravitational potential that gives most of the predictions that should be important to space science.

\subsection{Comparison Between Theory and Experiment}

Several comparisons were given to compare the predictions of these new gauge potentials with experiments that have already yielded data. Some of these comparisons produced predictions that virtually duplicated the predictions of other theories such as 
general relativity in the advance of the planetary perihelion. Others produced predictions at variance with other theories such as the prediction that the universe need not have started with a big bang. Yet even this prediction of variance came with the support for an explanation of early on exponential expansion that has been proposed without adequate previous support.

\subsubsection{Red Shifts}

The approach to the prediction of red shifts in the DT uses the assumption of isentropic propagation of light. This brings both the time and the gravitational effects of the source and the point of reception of the light into the picture. Thus, if the point of reception is above the Earth's surface, such as at the height of the Hubble telescope the measured red shift would be different from that measured at the Earth's surface. This shows up in the red shift prediction formula. However, it is the policy of the scientists at the Hubble telescope to correct the data to reflect the value of the red shift at Earth's surface thereby removing to potential to observe this prediction.

In spite of the scientific community not knowing of this point of reception prediction, there is also the exponential dependence upon both time and the gravitational potential that changes the red shift predictions. These exponential effects show that bodies with large gravitational potentials, say quasars, may have larger red shifts than time or distance alone would predict. Thus there need be no "queerness" associated with quasars since the large red shift occurs due to the exponential gravitational potential rather than extreme distances.

\subsubsection{Einstein's Field Equations}

Einstein's field equations show up in the DT as well as in Einstein's general theory of relativity. However, they show up for a very different reason and this changes interpretations that relate to the field equations. For example, because Einstein sought to find a means of describing dynamics as geodesics in a curved space rather than being due to the action of a force, he interpreted the resulting field equations to imply a curvature to the universe. On the other hand, the DT uses the 1st Law, the Conservation of Energy, in which to write the assumed universe geometry and then applies the 2nd Law to obtain a solution manifold for isolated systems. This solution manifold may then be used to obtain solutions, but does not impose any curvature upon the universe geometry. Then in this solution manifold when the conservation of mass is imposed the result is to reduce the five variable manifold of the solution manifold to a four variable hyper-surface of space and time upon with the field equations of Einstein describe the curvature of the solution hyper-surface.

The results of the imposition of conservation of mass upon the fundamental laws thus produce a set of equations as the Einstein field equations, yet they are obtained in a very different manner and the interpretations of general relativity cannot be applied. In particular, the interpretation that we live in a curved space has not foundation. This is because the curvature described by the field equations of the DT applies only to the solution hyper-surface imbedded into the solution manifold by the conservation of mass. This provides no basis for an interpretation of a curved universe.

Further, since Einstein obtained his field equations and then sought solutions to these equations in order to seek the dynamics due to gravity he found it necessary to determine solutions to a complicated system of equations. This allows for various solutions depending upon the restrictive assumptions thought to be applied. On the other hand, the DT yields the force law due to the negative gradient of the gravitational gauge potential. This potential and the resulting force establish the field equations and, therefore, no solutions need to be sought. Rather, the dynamics might be much easier to obtain using the five variable forces equations while assuming that mass is conserved.

\subsubsection{Dark Matter}

Dark Matter has yet to find an acceptable explanation among scientists. In the DT the time dependence of the gravitational field provides an explanation in the difference in between the tangential velocities in the arms of the spiral galaxies using the Newtonian time independent gravity and the time dependent gravity of the DT. The reason that this time dependence produces a different in the prediction of the tangential velocities is due to the time delay of the propagation of changes in the gravitational field that may only travel at the speed of light. This means that the gravitational field changes of the mass at the center of the galaxy do not arrive at the point of interest in the galactic arms until much later in time. Thus, the stars in the arms are reacting to the stronger gravitation of the central mass that it had when it departed from the center. This means that the tangential velocities may be expected to remain higher than they would if using the time independent Newtonian gravitational analysis. 


\subsubsection{Dark Energy}

The time dependence of the gravitational field also enters into the analysis of the data that argues for Dark Energy. This is a somewhat long and difficult analysis, but not any more difficult than is required using the cosmological constant of Einstein's general relativity. However, an overriding consideration is the influence of the time dependence of the gravitation field upon the Chandrasekhar limiting mass. This influence is to cause the limiting mass to change in time thereby effectively removing its use in the assumption that the Type Ia supernovas will make good standard candles due to their assumed constant luminosity.

Both of these differences should not stop any analysis of the data since the time dependence of the gravitational field is specified.

\subsubsection{Cosmic Background Radiation}

The cosmic background radiation is currently thought to be the result of the cooling down of the hot universe causes by the hot big bang. The DT though shows that a big bang need not be the only answer to how the universe developed in time. If there were no big bang, then what would account for the cosmic background radiation?

In the DT the radiation pressure of is always a little less than the radiation energy and is a function of the radiation frequency, or wavelength. Thus, in empty space where there exist no inertial mass to establish something to push against no pressure should be developed. In this case of zero pressure there will still be a required non-zero radiation energy density. This required remaining energy density is the source of the cosmic background radiation.

\subsubsection{Alternate Communications}

The derivation of the gauge field equations in a five variable Weyl manifold produces the eight inductively coupled differential equations that form an extended set of Maxwell-like equations. This set of differential equations lead to wave equations in the five variable manifold. There are ten components to these waves and these ten components form two virtually independent wave types. The ten components are the three components of the electric vector, three components of the magnetic vector, three components of the gravitational vector and the scalar gravitational potential.

The two types of waves may be called a transverse wave and a non-transverses wave. The transverse wave has only vector components all of which are directed transverse to the direction of propagation. The non- transverse wave is composed of an electric and a gravitational vector plus the scalar gravitational potential. This means the addition of scalar component might make it confusing to call this wave type a longitudinal wave and, rather, the title of non-transverse seems more appropriate.

Given two types of waves predicted several questions arise. One of the first questions might well be that of another possible means of passing information through an alternate form of communications based upon the nontransverse waves that are independent of the transverse waves. This would allow for an increase in communication methods without the increase in interference with nearby frequencies now becoming a problem in transverse wave communication systems. But how might this be done if the wave types are independent?

When the boundary conditions are investigated for an interface between materials, these show that there exist a means of converting some of the energy in a purely transverse wave into non-transverse wave energy. This would constitute a non-transverse wave sending antenna. Also, these exists a means of using the boundary conditions of an interface to convert some ot the energy of a purely non-transverse wave into a transverse wave thereby making a non-transverse wave receiving antenna.

\subsubsection{Earth Flyby Anomalies}

Earth flyby anomalies involve an interesting interaction of the inductively coupled electromagnetic and gravitational fields. First, the inductive coupling predicts that the spinning Earth must have a magnetic moment. Secondly, it predicts that the mass of the satellite flying by will have an effective electric charge that will interact with the Earth's magnetic field. The weakness of this effective charge accounts for the small anomaly while the interaction with between the effective electric charge of the satellite and the Earth's magnetic field accounts for the appearance of the declination in the prediction of the anomaly.

\section{REFERENCES}

Caratheodory, C., 1909. Untersuchungen über die Grundlagen der Thermodynamik. Math. Ann., 67: 355-386. DOI: 10.1007/BF01450409

London, F., 1927. Quantum Mechanical Interpretation of Weyl's Theory. Zeit F. Phys.

Weyl, H., 1952. Space, Time, Matter. 1st Edn., Courier Dover Publications, New York, ISBN-10: 0486602672, pp: 330. 
Williams, P. E., 2010c. The Dynamic Theory-A New View of Space-Time-Matter. 1st Edn., Williams Research, ISBN-10: 0615447112, pp: 172-194.

Williams, P. E., 2010d. The Dynamic Theory-A New View of Space-Time-Matter. 1st Edn., Williams Research, ISBN-10: 0615447112, pp: 175-176.

Williams, P. E., 2010e. The Dynamic Theory-A New View of Space-Time-Matter. 1st Edn., Williams Research, ISBN-10: 0615447112, pp: 296-303.

Williams, P. E., 2010f. The Dynamic Theory-A New View of Space-Time-Matter. 1st Edn., Williams Research, ISBN-10: 0615447112, pp: 314-319.

Williams, P. E., 2010h. The Dynamic Theory-A New View of Space-Time-Matter, 1st Edn., Williams Research, ISBN-10: 0615447112, pp: 303-309.

Williams, P. E., 2010i. The Dynamic Theory-A New View of Space-Time-Matter. 1st Edn.,Williams Research, ISBN-10: 0615447112, pp: 319-325.

Williams, P. E., 2010j. The Dynamic Theory-A New View of Space-Time-Matter. 1st Edn., Williams Research, ISBN-10: 0615447112, pp: 325-337.

Williams, P. E., 2010k. The Dynamic Theory-A New View of Space-Time-Matter. 1st Edn., Williams Research, ISBN-10: 0615447112, pp: 344-356.

Williams, P. E., 2010m. The Dynamic Theory-A New View of Space-Time-Matter. 1st Edn., Williams Research, ISBN-10: 0615447112, pp: 358-389.

Williams, P. E., 2010n. The Dynamic Theory-A New View of Space-Time-Matter, 1st Edn., Williams Research, ISBN-10: 0615447112, pp: 456-457.
Williams, P. E., 2010o. The Dynamic Theory-A New View of Space-Time-Matter. 1st Edn., Williams Research, ISBN-10: 0615447112, pp: 292-296.

Williams, P.E., 1997. Thermodynamic basis for the constancy of the speed of light. Modern Phys. Lett. A, 12: 2725-2738. DOI: $10.1142 / \mathrm{S} 0217732397002867$

Williams, P.E., 2001. Mechanical entropy and its implications. Entropy, 3: 76-115. DOI: 10.3390/e3030076

Williams, P.E., 2010a. The Dynamic Theory-A New View of Space-Time-Matter. 1st Edn., Williams Research, ISBN-10: 0615447112, pp: 107-111.

Williams, P.E., 2010b. The Dynamic Theory-A New View of Space-Time-Matter. 1st Edn., Williams Research, ISBN-10: 0615447112, pp: 131-137.

Williams, P.E., 2010g. The Dynamic Theory-A New View of Space-Time-Matter. 1st Edn., Williams Research, ISBN-10: 0615447112, pp: 289-292.

Williams, P.E., 20101. The Dynamic Theory-A New View of Space-Time-Matter. 1st Edn., Williams Research, ISBN-01: 0615447112, pp: 344-356.

Williams, P.E., 2010p. The Dynamic Theory-A New View of Space-Time-Matter. 1st Edn., Williams Research, ISBN-10: 0615447112, pp: 277-281. 